\title{
Formation and distribution of point defects on a disclination line near a free nematic interface
}

\author{
Jordi Ignés-Mullol,* Jean Baudry, and Patrick Oswald ${ }^{\dagger}$ \\ École Normale Supérieure de Lyon, Laboratoire de Physique, 46 Allée d'Italie, 69364 Lyon Cedex 07, France
}

(Received 1 October 2000; published 20 February 2001)

\begin{abstract}
Point defects of opposite signs can alternately nucleate on the $-1 / 2$ disclination line that forms near the free surface of a confined nematic liquid crystal. We show the existence of metastable configurations consisting of periodic repetitions of such defects. These configurations are characterized by a minimal interdefect spacing that is seen to depend on sample thickness and on an applied electric field. The time evolution of the defect distribution suggests that the defects attract at small distances and repel at large distances.
\end{abstract}

DOI: 10.1103/PhysRevE.63.031701

PACS number(s): 61.30.Jf, 61.30.Gd

\section{INTRODUCTION}

When a cylindrical cavity treated for homeotropic anchoring is filled with a nematic liquid crystal, a +1 -disclination line forms along its axis [1]. For energetic reasons, this line escapes [2,3] along the cylinder axis and radial $(R)$ and hyperbolic $(H)$ point defects can form. These defects were first observed by optical microscopy in capillary tubes of large diameters (more than 1 micrometer) [4-6] and more recently by nuclear magnetic resonance (NMR) in submicrometersize cavities [7]. Metastable configurations consisting of periodic repetitions of radial and hyperbolic defects (the socalled escaped-radial point defect, or ERPD configurations) are generally observed. Recent efforts have been made to understand the nature of the interaction between these defects [7-11]. All existing models and experimental observations agree that defects of opposite sign that are closer than a characteristic distance (proportional to the cylinder radius) attract and, eventually, annihilate. On the other hand, the interaction between defects placed beyond that characteristic distance is a matter of controversy. Peroli et al. [10-12] predict the existence of a finite cutoff distance $d_{c}$ below which defects attract and collapse and beyond which defects totally ignore each other. Recent experiments, performed in cylinders with diameters in excess of $100 \mu \mathrm{m}$ seem to be well described with this model [11]. On the other hand, Crawford et al. $[7,13,8]$ propose a model in which defects attract and collapse when they are placed at a distance less than $L_{0}$ and repel for distances larger than $L_{0}$. The resulting expansion of an array of defects would be pinned by the anchoring at the edges of the capillary tube, resulting in a metastable distribution with a well defined spacing between neighboring defects. NMR experiments performed in submicron cavities appear to be well described by this model. The difference in the capillary diameter in both types of experiments described above should not account for the discrepancies, since the only characteristic length scale in this problem is the tube diameter (as long as it is large compared with the core of the disclination). It is likely that anchoring effects on the capillary walls, and difficulties in the generation, control, and

\footnotetext{
* Present address: Departamento de Química Física, Universitat de Barcelona, Martíi Franquès 1, E-08028, Barcelona, Spain.

${ }^{\dagger}$ Electronic address: patrick.oswald@ens-lyon.fr
}

observation of defect distributions are at the source of the discrepancies.

In this paper, we propose to tackle a similar problem in a different geometry that allows us to visualize the defects in the microscope and to control both their initial and final densities. We recall that the problem of coarsening dynamics of topological defects already attracted much interest in the field of liquid crystals, but most experiments were performed in two [14] and three [15] dimensions.

The starting point of the experiments reported here is the formation of point defects on disclination lines of semiinteger ranks $[16,17]$. Such a line can appear near the sides of a nematic sample confined between two glass plates when the molecules are homeotropically anchored to both the glass and the free surface [Fig. 1(a)]. In thin samples (of thickness $b<100 \mu \mathrm{m})$, the free surface has the shape of a circular meniscus of radius of curvature $R>b / 2$. If the anchoring energy $W$ of the molecules at the free surface is large, i.e., if $W R / K \gg 1$ where $K$ is the bend elastic modulus, a disclination line forms at a well defined distance of the meniscus (it being agreed that the anchoring energy on the glass is very large in our experiment and can be considered as infinite). A detailed study of the conditions that lead to the formation of the disclination line, and the dependence of its equilibrium position on the different material and geometrical parameters has been the objective of a previous publication [16]. In that work, a model, which considered the structure of the director field to be both translationally invariant along the free interface and contained in a plane perpendicular to the free interface, was shown to give quantitatively accurate predictions on the position of the disclination line. Experimentally, however, it was clear that the structure of the director field in the vicinity of the disclination line had a three-dimensional nature. The escaped component of the director field (parallel to the interface and ignored by the planar model) is revealed by the shading that can be noticed when the sample is observed between partially crossed polarizers [Fig. 1(b)]. Moreover, point defects form along the disclination line, distorting the director field in a clearly three-dimensional fashion, and breaking the translational invariance. A more realistic, threedimensional model was used to compute the escaped component of the director field in the vicinity of the disclination line, and it was shown that the magnitude of that component increases as the core of the disclination is approached [Fig. $1(\mathrm{c})]$. If the structure of the director field is translationally 


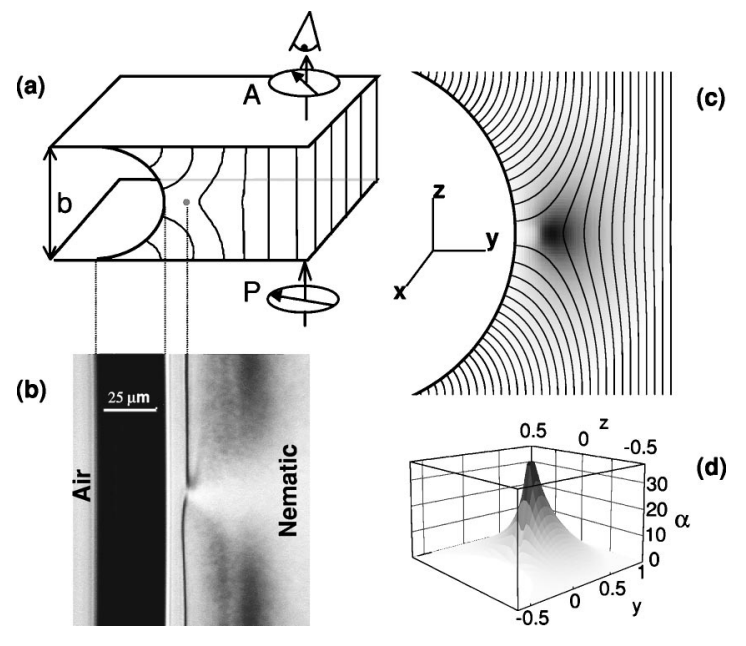

FIG. 1. (a) A disclination line forms in the vicinity of the free interface of a nematic liquid crystal confined between two plates with a uniform spacing $(b)$ between them and treated to induce a homeotropic anchoring. (b) Top view of an actual sample ( $b$ $=100 \mu \mathrm{m})$ when observed with a polarizing microscope with the polarizer $(P)$ and the analyzer $(A)$ partially crossed. No light is transmitted through the curved $N$ /air interface, resulting in a black meniscus region. The homeotropic structure of the director field is distorted from the homeotropic configuration in the vicinity of the interface.The translational symmetry along the meniscus is broken by the presence of a point defect. (c) Calculated projection of the director field on the $Y Z$ plane. The director field is tangent to the field lines (solid lines). The intensity of the gray shading is an encoding for the magnitude of the escaped component of the director field along the interface ( $X$ component). The corresponding angle $(\alpha)$ between the director field and the $Y Z$ plane is shown in (d). Lengths are in units of the cell gap.

invariant along the disclination line, it is clear that there will be a twofold degeneracy in the orientation of the escaped component, since the transformation $\alpha \rightarrow-\alpha$ results in an energetically equivalent configuration. Upon formation of the confined nematic phase, a local orientation of the escaped component of the director field must be chosen. When two regions where the escaped component of the director field has opposed orientations meet, a point defect structure forms. The point defects interact with each other, and that results in the rearrangement of their distribution along the disclination line, with the possibility of annihilation of pairs of defects and the formation of a well defined steady state distance between neighboring defects.

The goal of this paper is to study the formation of these defect distributions, since they may reveal information about the interaction between neighboring defects and, ultimately, about the structure of the director field near these complex structures. This paper is structured as follows. In Sec. II the details of the experimental procedures are described. In Sec. III the distribution of point defects is described in the absence of external field. The effect of an electric field in the structure of the nematic phase near the meniscus, as well as its use to control the distribution of defects is discussed in Sec. IV. In Sec. V the dynamics that leads to the formation of the defect distribution is discussed. A simple model, which suggests the existence of a net interdefect repulsion as the stabilizing mechanism, is presented in Sec. VI.

\section{EXPERIMENTAL PROCEDURE}

Our samples consisted of two glass plates $1 \mathrm{~mm}$ thick, $2 \times 2 \mathrm{~cm}^{2}$ of surface placed parallel to each other with either nickel wires or nylon threads setting the uniform spacing [see Fig. 1(a) for the sketch of a sample cell]. Values for the spacing were in the range $10 \mu \mathrm{m} \leqslant b \leqslant 220 \mu \mathrm{m}$. The indiumtin-oxide (ItO) coating of the glass plates allowed the application of an electric field across the sample. The glass plates were treated chemically in order to induce a strong hometropic anchoring of the nematic phase. After being throughly cleaned, the plates were dipped into a $0.002 \%$ aqueous solution of CTAB (hexadecyl trimethyl ammonium bromide) for a few minutes, then lifted slowly out of the solution. The cells were partially filled with 8CB (4- $n$ octylcyanobiphenyl, K24 from Merck) by capillarity. This generated the free nematic interface that was the object of our observations. We assume that the nematic-air meniscus has a semicircular cross section. This allowed us to estimate the contact angle at the glass plates to be close to $10^{\circ}$ [16]. The samples were kept at $T=36.5^{\circ} \mathrm{C}$, a temperature that is in the nematic phase range for $8 \mathrm{CB}$. In equilibrium, the nematic phase is homeotropic away from the open interface, and a disclination line forms in the vicinity of that interface. We have observed that, unavoidably, point defects appear along the disclination line. The number of defects seems to depend strongly on the history of the formation of the disclination line. For instance, if the cell is kept at a temperature in the nematic range at all times (including when it is first filled) we observe that, initially, the nematic phase is in a nonhomeotropic, unstable configuration. Regions with homeotropic orientation nucleate quickly and grow towards the open end with a speed that decreases with increasing sample thickness. This leads to the slow formation of a disclination line (it takes some seconds for the thinnest samples, and several minutes for the thickest ones). Along this disclination line we observe a number of defects, but they are sparsely distributed. On the other hand, if the formation of the disclination line is preceded by a sudden temperature quench it is possible to generate a dense distribution of defects. For instance, by cooling the isotropic phase, one generates a randomly oriented nematic phase, which is subsequently aligned by the anchoring imposed by the glass walls. One can also heat up the smectic-A phase, in which there is no defect when the sample is oriented homeotropically. In this case, a disclination line forms at the transition in the nematic phase providing that the anchoring torque on the free nematic interface is larger than the elastic torque required to accomodate the favored anchoring angle at the interface. In both cases, regardless of the initial state, the transient leading to the final homeotropic nematic configuration involves the formation of a disclination line through a nucleation process. The line nucleates simultaneously in different locations along the free interface. As we explained above, there is a twofold degeneracy in the orientation of the escaped component of the director field in the vicinity of the disclination line. Therefore, regions with opposed orientations can nucleate simultaneously. We believe 


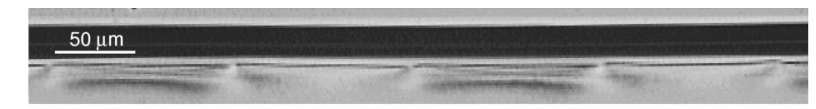

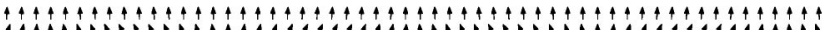

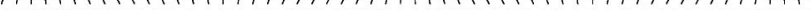
सी $\rightarrow$. $\because V_{1}$

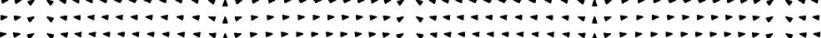

FIG. 2. Top: top view image of the region near the N/air interface in a $b=50 \mu \mathrm{m}$ sample observed between partially crossed polarizers [see Fig. 1(a)]. Note that the optical properties of the nematic phase alternate between consecutive point defects. A point defect is generated when two regions with opposed escaped orientations of the director field meet. Bottom: $X Y$ projection of the director field of the nematic phase in the presence of point defects near the midplane $(z=0)$ of the sample.

this is at the origin of the observed point defects (see Fig. 2). The visualization, between partially crossed polarizers, of alternating regions with different intensity of transmitted light between consecutive point defects along the disclination line is consistent with this description.

A final mechanism to control the formation or the distribution of point defects along the disclination line, which offers the greatest experimental control, consists on changing the magnitude of an electric field perpendicular to the glass plates (see Sec. IV). The electric field was introduced by applying a voltage between the two glass plates. The voltage was $\mathrm{AC}$ with a frequency $f=5 \mathrm{kHZ}$, in order to avoid triggering an electro-hydrodynamic instability due to ion migration. The choice of frequency did not have any observable effect on the results presented here.

\section{STEADY-STATE DISTRIBUTION OF DEFECTS IN THE ABSENCE OF EXTERNAL FIELDS}

Even though the defects are somewhat randomly distributed along the interface immediately after the formation of the disclination line, their distribution relaxes with time until the interdefect spacing is rather uniform (see Fig. 2). During this relaxation, it is possible to observe the annihilation of pairs of neighboring point defects. We have measured a thickness dependent critical interdefect separation below which neighboring defects appear to attract resulting in their collapse, which reduces the average wave number in the defect distribution. Defect distributions with average wavelength above the critical one are metastable (at least for periods of time up to several hours). An example of the dynamics that leads to the relaxation of an unstable defect distribution is shown in Sec. IV.

In Fig. 3 the measured values for the critical interdefect separation, $\lambda_{0}$, are presented as a function of sample thickness, $b$. Notice that $\lambda_{0}$ scales linearly with $b$, which is consistent with the observations published earlier [16] where, for material parameters and values of $b$ similar to the ones involved here, a condition of infinite anchoring on the free nematic interface was satisfied, and the only relevant length scale in the system affecting the position of the disclination line was $b$.

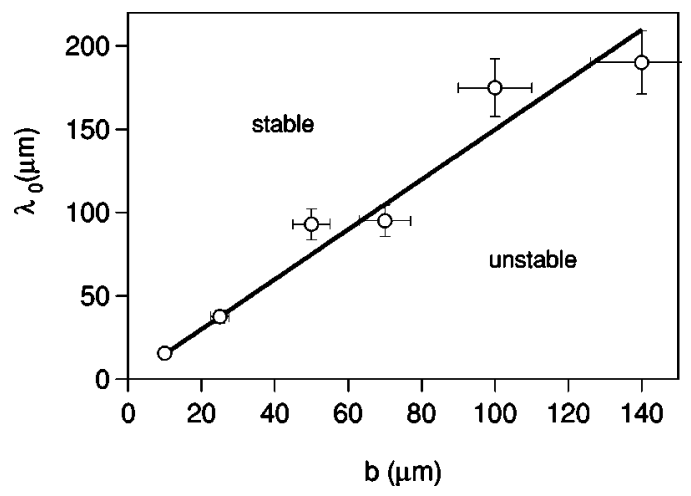

FIG. 3. Critical equilibrium wavelength (interdefect spacing) for the distribution of point defects along the disclination line, as a function of sample thickness. Distributions with $\lambda<\lambda_{0}$ are unstable and annihilation of some neighboring pairs will occur. Distributions with $\lambda \geqslant \lambda_{0}$ are stable. The critical wavelength appears to scale with sample thickness as $\lambda_{0}=(1.5 \pm 0.2) b$.

When performing observations for extended periods of time (several hours), defect distributions are not seen to collapse. Instead, there is a trend for the average spacing to increase slightly. A long string of defects is bounded by pinning points found along the disclination line. We also observe a slow drift of the defects (which seems to have no influence on the defect spacing). This is most likely due to small temperature gradients in the oven.

As explained above, the fact that the director field has a three-dimensional component along the disclination line is at the origin of the observed point defects. Therefore, the magnitude of the escaped component away from point defects must be directly related to the parameter $\lambda_{0}(b)$ reported above. A configuration with a smaller magnitude in the escaped component would, presumably, result in a tighter distribution of defects. On the other hand, the fact that a change in $b$ (always very large compared to the core radius of the disclination line) introduces a trivial scaling in the equilibrium interdefect distance suggests that the ratio $\lambda_{0}(b) / b$ and, therefore, the magnitude of the escaped component of the director field will be determined by the elasticity of the nematic phase and by the anchoring at the free interface and will be independent of the thickness of the sample (at least in the regime where the position of the disclination line is observed to scale linearly with the sample thickness). Since the goal of the current work is to study the structure of the director field near the free interface and the nature of the interaction between defects, we have sought to better understand the relationship between the interdefect distance and the escaped component of the director field.

In Sec. IV a description of how the distribution of defects can be carefully controlled by means of an external electric field is presented. The measurements are complemented with numerical estimations of the three-dimensional structure of the director field in the vicinity of the free interface.

\section{EFFECT OF AN ELECTRIC FIELD}

\section{A. Coupling with the structure of the director field}

An external electric field perpendicular to the cell plates favors the homeotropic orientation of the nematic molecules 


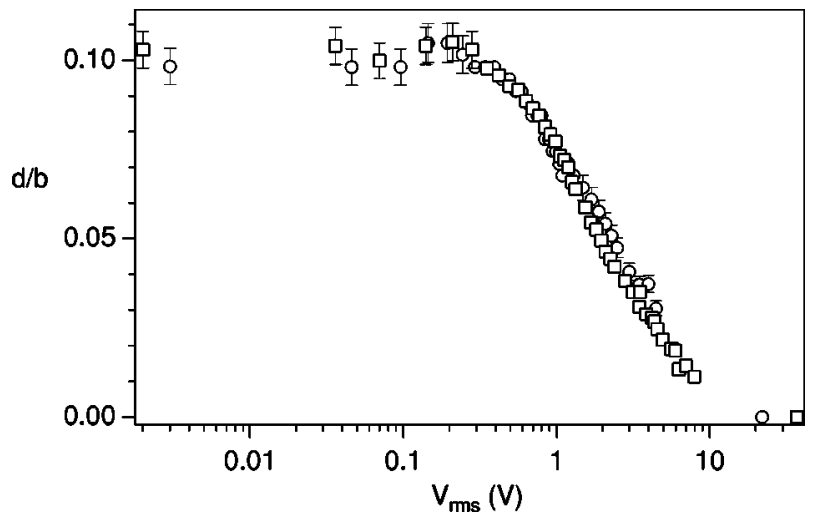

FIG. 4. Ratio of the distance between the disclination line and the tip of the N/air meniscus, $d$, to the sample thickness, $b$, as a function of the voltage across the glass plates due to the external electric field. Samples with gaps $b=70 \mu \mathrm{m}(\bigcirc)$ and $b=220 \mu \mathrm{m}$ ( $\square$ ) are reported. Note that only in the regime where the disclination line approaches zero is there an observable thickness dependence of the ratio $d / b$.

for a material with positive dielectric anisotropy $\varepsilon_{a}$, such as $8 \mathrm{CB}$. One expects the effect of the electric field to be strongest in the vicinity of the free interface, where the structure of the director field is further distorted from the homeotropic configuration by the presence of the curved meniscus. The electric field is applied by using ItO coated glass plates, which act as electrodes, to build the cells, as described in Sec. II.

The most immediately observable effect of the electric field is the alteration of the position of the disclination line (see Fig. 4). At low enough voltages, the disclination line remains at the $V=0$ equilibrium position. For applied voltages above a thickness-independent threshold $\left(V_{t h}\right)$, however, the disclination line starts to approach the meniscus. Notice that for the range of voltages presented on Fig. 4, the ratio $d / b$ is independent of sample thickness, which suggests that a condition of strong anchoring at the interface prevails. Dimensional analysis suggests that the position of the disclination line is given by $d / b=f\left(\varepsilon_{0} \varepsilon_{a} V^{2} / K\right.$ ) (see the Appendix), which is compatible with experimental observations when different liquid crystals are used. The experiments with $8 \mathrm{CB}$ reported on Fig. 4 show $V_{t h} \simeq 0.4 \mathrm{~V}$. Based upon the expressions above, one would expect a dependence of the form $V_{t h} \propto\left[K /\left(\varepsilon_{0} \varepsilon_{a}\right)\right]^{1 / 2}$. With typical values $K \approx 5 \times 10^{-7}$ dyn and $\varepsilon_{a} \approx 10$, one calculates $V_{t h} \approx 0.24 \mathrm{~V}$ in qualitative agreement with the experimental value.

Along with the decrease of $d$, the distortion due to the escaped component of the director field is also observed to decrease as the applied voltage increases. At a thicknessdependent value of the applied voltage, $V_{c}$, the disclination line appears to collapse (see the Appendix). Even though the line is not directly observable for voltages close to but below $V_{c}$, the value of $V_{c}$ can be estimated quite accurately by observing the distribution of point defects. Indeed, if the applied voltage is decreased before reaching $V_{c}$, the threedimensional structure of the director field is remembered, and the distribution of defects remains unchanged. However, if $V$ is increased beyond $V_{c}$, a subsequent decrease in the

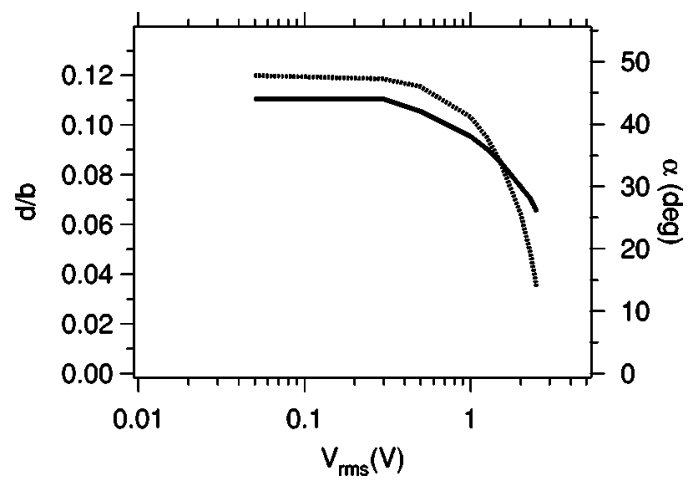

FIG. 5. Numerical calculation of the position of the disclination line (solid line) and magnitude of the maximum escape angle (dashed line) as a function of the applied voltage.

applied voltage generates a different distribution of defects, thus suggesting that the disclination line had disappeared.

\section{B. Model}

The effect of the electric field both on the position of the disclination line and on the escaped component of the director field can be qualitatively reproduced numerically. We have used the full Landau-Ginzburg-de Gennes tensorial expansion for the elastic free energy density in power series of the quadrupolar order parameter, and considered a rigid homeotropic anchoring of the nematic molecules on the meniscus $[16,18]$. As an important simplification, we have assumed the electric field to be uniform inside the sample. Solving the boundary-value problem which results after minimizing the free energy functional, the results are as presented in Fig. 5. The numerical solution for the position of the disclination line reproduces qualitatively the features found experimentally. In particular, it predicts the plateau at low voltages and the roughly exponential decrease in $d$ for $V>V_{t h}$. In fact, the numerical estimations can be quantitatively brought on top of the experimental data in Fig. 4 remarkably well by considering an effective electric field about $40 \%$ smaller than the real one. Since the three-dimensional structure of the director field is computed, we obtain also an estimation for the changes in the maximum tilt angle $\alpha$ of the director with respect to the $Y Z$ plane (Fig. 2) as the electric field is increased. Since $\alpha$ is not directly measurable experimentally, we will have to rely on the numerical estimations as a first approximation for it. The validity of the computations can be justified by how closely they reproduce the observed evolution of the disclination line and, therefore, the structure of the director field. The model predicts the escaped component of the director field to collapse much faster with increasing electric field than the disclination line does.

\section{Distribution of defects in the presence of an electric field}

Similarly to what was measured in the absence of an electric field (Sec. III), an applied voltage-dependent equilibrium wavelength for the defect distribution, $\lambda_{0}(V, b)$, is observed. A distribution that packs defects tighter, on average, than $\lambda_{0}(V, b)$ appears to be unstable. Pairs of neighboring defects approach and collapse, increasing the average wavelength of 


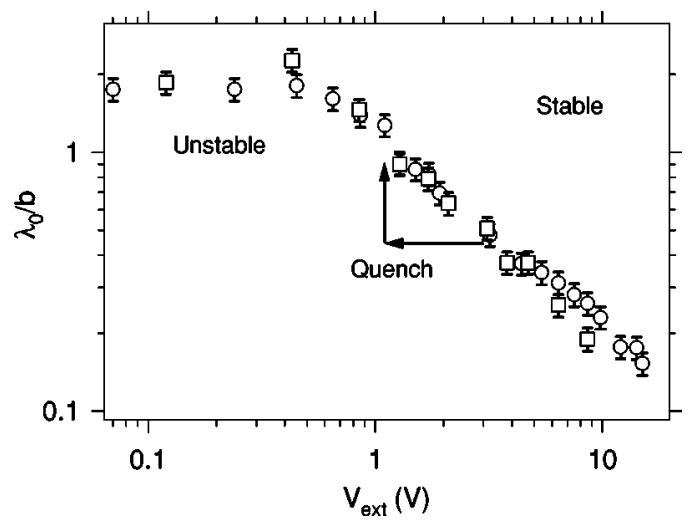

FIG. 6. Average wavelength of marginal stability of the defect distribution $\left(\lambda_{0}\right)$ in the presence of an electric field. The data is plotted as a ratio $\lambda_{0}$ to $b$ vs rms applied voltage using a log-log plot. Note that no sample thickness dependence, other than a trivial scaling, is observed. Data corresponding to two sample thicknesses are reported: $b=50 \mu \mathrm{m}(\square)$ and $b=100 \mu \mathrm{m}(\bigcirc)$. Quenching the applied voltage into the unstable region triggers a dynamical rearrangement of the distribution, ending in a new configuration with a larger average spacing.

the distribution. Along with this process, one also observes a tendency for the defect distribution to rearrange so that there is a quite uniform value for the interdefect spacing (see Sec. $\mathrm{V}$ for a more detailed analysis of the dynamics leading to the formation of the distribution of defects). On the other hand, a distribution whose average interdefect spacing is larger than $\lambda_{0}(V, b)$ appears to be stable for extended periods of time (they can be observed for minutes or even hours). In Fig. 6, the measurements for $\lambda_{0}(V, b)$ with two different sample thicknesses are reported by plotting the ratio $\lambda_{0}(V, b) / b$. Note that, consistently with the observations reported in Figs. 3 and 4, no sample thickness dependence is observed in that ratio, within our optical resolution. Therefore, the thickness dependence in $\lambda_{0}(V, b)$ can be trivially expressed as $\lambda_{0}(V, b)=b \Lambda_{0}(V)$ where $\Lambda$ denotes the dimensionless wavelength. For $V<0.5 \mathrm{~V}, \Lambda_{0}$ is roughly constant, and equal to the value measured in the absence of an electric field (see Fig. 3). As $V$ is increased beyond $0.5 \mathrm{~V}, \Lambda_{0}$ starts to decrease following roughly a power law. It is interesting to observe that the effect of the electric field on the distribution of defects starts to be noticeable at about the same voltage ( $V$ $\simeq 0.5 \mathrm{~V}$ ) that on the position of the disclination line (see Sec. IV A). This is consistent with the predictions of our numerical model (Fig. 5), which shows that both the position of the disclination line and the escaped component of the director field (which we believe to be intimately related to the defect distribution) start to vary from their $V=0$ value at roughly the same applied voltage. $\Lambda_{0}$ appears to decrease faster than the position of the disclination line with increasing $V$, consistently with the numerical result that the escape angle falls faster than $d$.

In Sec. $\mathrm{V}$ the process that leads to the formation of the equilibrium defect distribution is addressed. It is described in two steps: first, the average $\lambda$ evolves towards the steady state value. Next, the width (dispersion) of the defect distribution decreases steadily.

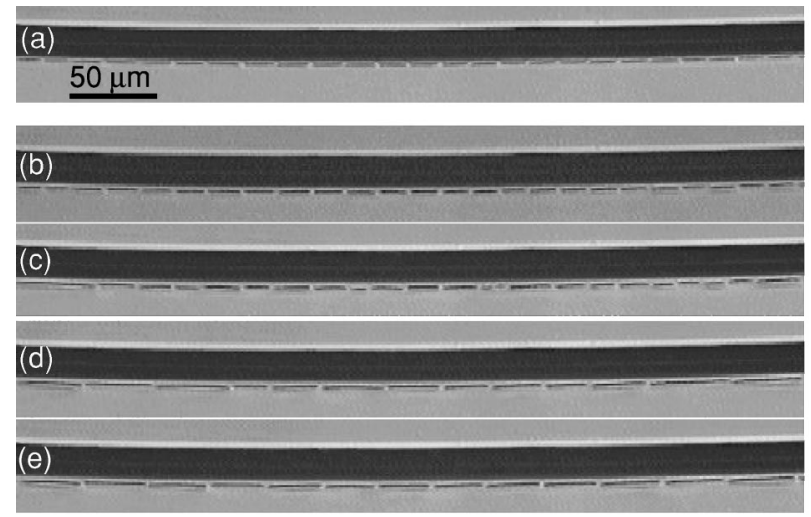

FIG. 7. Top view of a sample during the reorganization of the defect distribution after a quench of the electric field into an unstable configuration. (a) Initial equilibrium distribution at $V_{1}$ $=2.26 \mathrm{~V}$. (b) The sample is suddenly quenched to $V_{2}=0.86 \mathrm{~V}$. (c) $20 \mathrm{~s}$ after the quench, the average defect spacing is reduced by annihilation of pairs of defects. (d) $70 \mathrm{~s}$ after the quench, the equilibrium wavelength at $V_{2}$ has already been achieved, but there is large dispersion. (e) $240 \mathrm{~s}$ after the quench, the distribution is sharply peaked at the equilibrium wavelength. The cell gap is $b$ $=50 \mu \mathrm{m}$.

\section{DYNAMICS OF THE FORMATION OF THE STEADY-STATE DISTRIBUTION OF DEFECTS}

The use of an applied electric field allows not only to tune the defect distribution, but also to explore the dynamics that leads to the formation of the distribution. It is possible to prepare a string of defects with an arbitrary wavelength by applying a voltage as seen in Fig. 4. After a transient time, which decreases with increasing $V$, a rather uniform defect distribution is obtained. It remains stable for an extended period of time. An increase in $V$ will not alter the stability of the distribution. A quench in the applied voltage, however, will make the distribution unstable (its wavelength will be too short for the lower voltage) and will trigger a rearrangement of the defects that will involve some annihilations.

The goal is to study how the distribution of point defects changes when it is made unstable by a sudden quench in the electric field. In order to perform a statistical analysis, we proceed by repeatedly measuring how the wavelength distribution changes with time after a quench between two given voltages. First, we prepare a stable configuration, i.e., a distribution of defects sharply peaked at $b \Lambda_{0}\left(V_{1}\right)$. In order to obtain uncorrelated initial distributions, the disclination line is collapsed between repetitions of the experiment. That is achieved by applying a voltage $V>V_{c}$ (see the Appendix) prior to setting the voltage to the initial value $V_{1}$. Next, five minutes are allowed for the initial distribution to reach the equilibrium configuration before quenching the voltage to a lower value $V_{2}$.

Figure 7 shows pictures of a defect distribution while it is relaxing from an initial voltage to a final voltage after a quench. In Fig. 7(a), an initial, equilibrium configuration of defects under an applied voltage $V_{1}=2.26 \mathrm{~V}$ is prepared. Then, the sample is suddenly quenched into a smaller applied voltage $V_{2}=0.86 \mathrm{~V}$ [Fig. 7(b)]. The system, now un- 


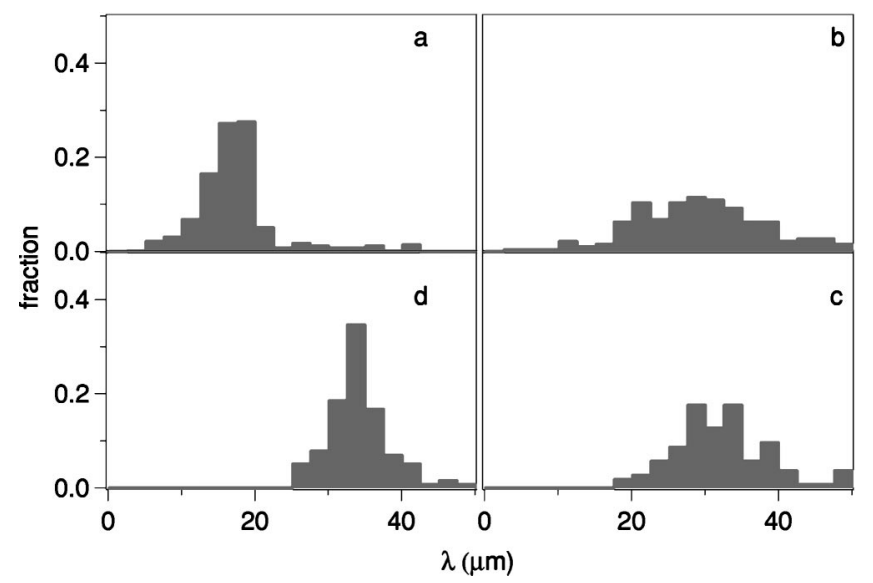

FIG. 8. Evolution of the distribution of point defects after quenching a $b=50 \mu \mathrm{m}$ pure $8 \mathrm{CB}$ sample. Data from 12 experimental realizations are combined. At $t=0$, the voltage is quenched from $V_{1}=2.56 \mathrm{~V}$ into $V_{2}=1.06 \mathrm{~V}$. The histogram of the wavelength distribution is shown at (a) $10 \mathrm{~s}$, (b) $40 \mathrm{~s}$, (c) $80 \mathrm{~s}$, and (d) $540 \mathrm{~s}$ after the quench.

stable, will evolve towards a new equilibrium distribution with a smaller wave number (larger wavelength) in the defect distribution. At early times, this is mainly achieved by annihilation of pairs of defects [Fig. 7(c)]. When the average wave number is close to $b \Lambda_{0}\left(V_{2}\right)$ defect annihilation stops. The distribution of interdefect spacings, however, has a large dispersion, and many different values are observed in the sample [Fig. 7(d)]. The next stage in the evolution towards the final equilibrium configuration is the relaxation of this distribution, reducing its dispersion and sharply centering it about its new mean, $b \Lambda_{0}\left(V_{2}\right)$ [Fig. 7(e)]. In order to gain some more understanding about the dynamics that leads to the reorganization of an unstable distribution, the evolution of the defect distribution after a quench in the applied electric field is analyzed statistically. Since only a relatively small portion of a sample fits in the field of view at any given time, data from different independent experiments performed under the same conditions are combined. Note that this assumes that the evolution of the defect distribution for regions in a sample that are more than a few times the average wavelength apart are uncorrelated. This seems to qualitatively agree with our observations. In Figs. 8 and 9 the results corresponding to a series of experiments performed with a $50 \mu \mathrm{m}$ thick, pure $8 \mathrm{CB}$ sample are presented. The process is the same as that in the images in Fig. 7. It begins with the preparation of an equilibrium defect distribution at $V_{1}$ $=2.56 \mathrm{~V}$. That is, the distribution of defects is narrow and centered about $\lambda_{\text {ave }} \simeq b \Lambda_{0}\left(V_{1}\right)$. At $t=0$, the applied voltage is quenched into $V_{2}=1.06 \mathrm{~V}$. This quench is faster than any observable relaxation time in the distribution. Combining data from different experiments, we have extracted the histogram for the distribution of values of the interdefect spacing (wavelength) as a function of the elapsed time since the quench. In Fig. 8, the histograms corresponding to four stages in the evolution are presented. Notice that $10 \mathrm{~s}$ after the quench the distribution has already started to reorganize. The peak is still close to the initial value (just a few annihi-

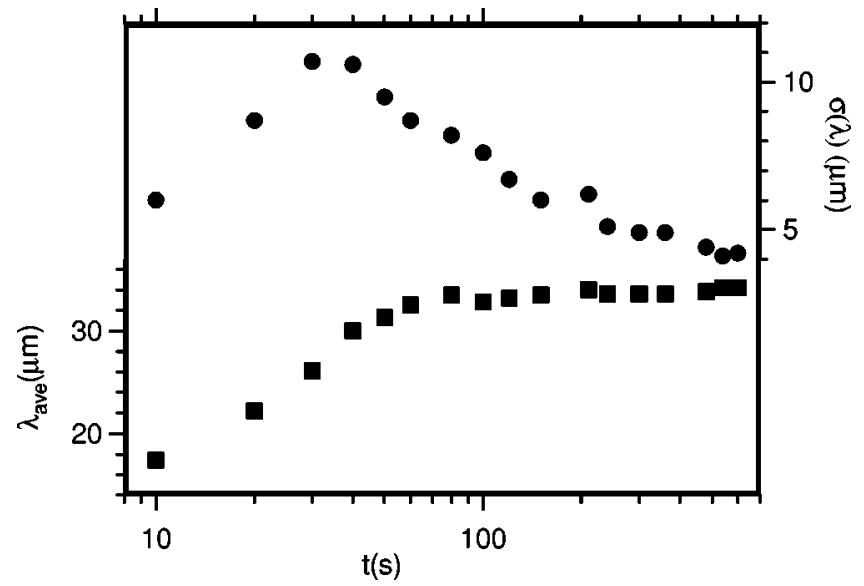

FIG. 9. Evolution of the mean, $\lambda_{\text {ave }}(\boldsymbol{\square})$, and the standard deviation, $\sigma(\lambda)(\bigcirc)$, of the spacing of the defect distribution vs the time since the electric field quench, for the same data presented in Fig. 8.

lations have taken place) but the width of the distribution has started to increase. After $40 \mathrm{~s}$, an important number of annihilations of pairs of defects have occurred. As a result, the dispersion in the distribution has increased sharply. After 40 $\mathrm{s}$, the position of the peak seems almost stabilized, and the dispersion starts to decrease. Several minutes after the quench $(540 \mathrm{~s})$, the defect distribution is sharply centered about a new equilibrium value, $\lambda_{\text {ave }} \simeq b \Lambda_{0}\left(V_{2}\right)$. In Fig. 9 the time evolutions of the mean and standard deviations of the defect distribution are plotted. Notice that, while annihilation of defects effectively brings the average wavelength within a few percent of the final equilibrium value in less than $60 \mathrm{~s}$, the width of the distribution is decreasing roughly exponentially with time, even $600 \mathrm{~s}$ after the quench. Similar measurements have been performed with smaller values of $V_{2}$ (using the same starting voltage $V_{1}$ ). The relaxation of the defect distribution is slower with decreasing $V_{2}$.

\section{MODEL FOR THE INTERACTION BETWEEN DEFECTS}

In order to understand the observed formation and distribution of defects, a good model for the three-dimensional structure of the director field is required. Unfortunately, the structure of the director field in the vicinity of the point defects is probably rather complex. As an approximation, which will prove to give results that agree qualitatively with the experimental observations, we propose a simplified model for the structure of the director field. We will not attempt to obtain the configuration that minimizes the elastic energy in the system. Instead, we will use the model to study the effect that changes in the relative position of two point defects may have in the elastic energy of the system.

The three-dimensional director field in the vicinity of the $N$ /air meniscus and in the presence of a disclination line was calculated as shown in Fig. 1 and detailed in Sec. IV B with the assumption of a structure that is translationally invariant along the disclination line. Clearly, the presence of the point defects breaks that symmetry, as discussed above. The trans- 


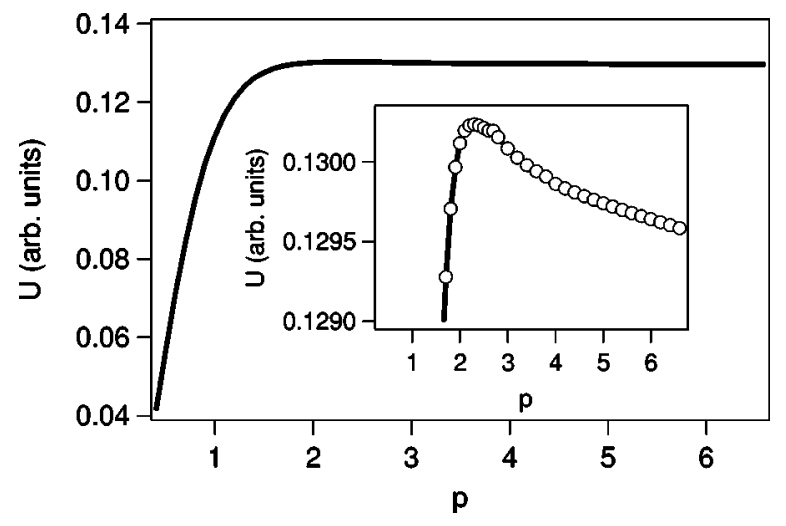

FIG. 10. Elastic energy $(U)$ associated with an array of point defects with a spacing $p$ (in units of the cell gap) between them. The energy of the translationally invariant escaped configuration is taken to be $U=0$, and that of the planar configuration is taken to be $U=1$. A value $B=1.5$ is chosen to characterize the extent of the domain walls, as described in the text. The inset shows a magnified detail of the energy curve that reveals the presence of a maximum.

lationally invariant structure of the director field is of the form $\mathbf{n}_{\mathbf{x}}=\sin [\alpha(y, z)]$ (escaped component, i.e., parallel to the disclination line, see Fig. 1), $\mathbf{n}_{\mathbf{y}}=\cos [\Phi(y, z)] \cos [\alpha(y, z)]$, and $\mathbf{n}_{\mathbf{z}}=\sin [\Phi(y, z)] \sin [\alpha(y, z)]$. The model we propose breaks the translational invariance along the disclination line by replacing the angle between the director field and the $X$ axis, $\alpha(y, z)$, with $\alpha(y, z) f(x)$, where $f(x)$ will be \pm 1 away from the point defects and will change between both values in a small region around the defect. The structure of the point defects is modeled as a domain wall, analogous to the distortions that a magnetic field introduces on a bulk nematic [19]. We use the form $f(x)=\tanh (B x)$, where $1 / B$ is the characteristic width of the wall. This expression models the region where the two opposed (and energetically equivalent) orientations of the escaped field meet. We observe that point defects have a spatial extent of the order of the cell gap, $b$. Therefore, values of $B$ close to 1 (in units of $b$ ) will be reasonable. The presence of two opposed point defects will be modeled using the expression

$$
f_{2}(x)=-1+\tanh \left[B\left(x+\frac{p}{2}\right)\right]-\tanh \left[B\left(x-\frac{p}{2}\right)\right],
$$

which represents two defects located at $x= \pm p / 2$ (therefore with a spacing $p$ between them). The elastic energy is calculated by integrating the expression

$$
\rho f=\frac{K}{2}\left[(\operatorname{div} \mathbf{n})^{2}+(\operatorname{curl} \mathbf{n})^{2}\right]+\frac{K_{2}-K}{2}(\mathbf{n} \cdot \operatorname{curl} \mathbf{n})^{2},
$$

where $K_{1}=K_{3}=2 K_{2}=K$ [20]. The integration is carried out in a volume that includes both defects and extends long enough along the $X$ axis that the presence of the defects has a negligible effect in the elastic energy in the boundaries of that interval. This region is also extended a distance $1.5 \mathrm{~b}$ behind the disclination line, where the director field is practically homeotropic $\left(\mathbf{n}_{\mathbf{z}}=1\right)$. In Fig. 10 the calculated energy as a function of the spacing between the two defects is shown. A value of $B=1.5$ has been used for the calculations reported here. For the range of spacings explored $(0<p$ $<10)$ the domain of integration spans the $X$-axis interval $-10<x<10$. The energy of a translationally invariant escaped configuration (no defects) has been chosen as the reference. The units of energy have been chosen so that the difference between the energy of a planar configuration $\left(\mathbf{n}_{\mathbf{x}}\right.$ $=0$ ) and the reference is 1 . The calculations show, in accordance with the experimental observations, that defects attract at short distances, with the energy converging towards the reference value. As the spacing between defects increases the elastic energy approaches asymtotically a value that would correspond to the reference value plus twice the energy associated with an isolated point defect. A magnification of the calculated energy curve (see inset in Fig. 10) shows the interesting fact that there is a maximum in that curve (at $p$ $\simeq 2.3$ ). The model, therefore, predicts an attraction when the defects are closer than $p \simeq 2.3$ and a weak repulsion when they are further apart than $p \simeq 2.3$. This is consistent with the distribution of defects evolving towards a uniform spacing once the average spacing between defects is larger than a critical value, since that is the configuration where mechanical equilibrium will be achieved in the presence of a net interdefect repulsion. Another consequence of this net repulsion should be an expansion of the defect distribution with time. This would necessarily be a much slower process than the collapse of two neighboring defects, since it involves a collective motion of all the defects and very weak repulsive interactions. This is what we observed experimentally. Distribution of defects appear to be stable for extended periods of time but display a slight trend towards spreading, not collapsing, consistent with a net repulsion between neighboring defects. In addition, we observed a very slow translation of the distribution of defects outside of the field of view, which may be explained by the presence of a small temperature gradient along the meniscus.

The model presented here is likely to be insufficient to accurately describe the configuration of the director field as two defects are about to collapse. As the spacing between the defects increases, the details of the structure of the point defect are probably less crucial, and the model should be more accurate.

\section{CONCLUSION}

We have found a new experimental configuration that allows to easily control the nucleation and the dynamics of point defects along a disclination line. This experiment has the advantage to be much easier to perform than those in a circular capillary tube where ERPD configurations are not so easy to produce. In particular, the actual geometry has the advantage that an electric field can be easily applied to control the initial density of defects. The main disadvantage of our geometry is that the director field is much more difficult to calculate than in the axisymmetric case. On the other hand, the dynamics of the defect distribution suggests that the interactions between defects of opposite sign are the same as those observed in NMR experiments in submicron capillary tubes [7]. Indeed, the increase at short time of the 
width of the distribution while the average wavelength increases [21] shows that defects of opposite signs attract at short distance (less than typically $1.5 b$ ). By contrast, the saturation of the mean wavelength while the distribution becomes more and more peaked suggests that the defects repulse above some distance as radial and hyperbolic defects appear to do in submicron capillaries. On the other hand, the results described here are different from those obtained in experiments with thick capillary tubes [11], where a cutoff length appears to exist beyond which defects no longer interact while defects closer than $d_{c}$ attract and collapse. This would lead to a very disperse, stationary distribution of the distance between defects, which would be strongly dependent on the initial distribution of defects [22]. A simple model for the structure of the director field (Sec. VI) reproduces qualitatively the observed phenomena, including the short range attraction and the weak long range repulsion between neighboring defects. Work is currently under way to calculate the elastic interaction potential between defects of opposite sign and to study the dynamics of point defects distributions in the system described here using Monte Carlo simulations.

\section{ACKNOWLEDGMENTS}

This work was supported by the European Research Network Contract No. FMRX-CT96-0085. The authors thank Lubor Lejcek for helpful discussions and guidance in setting up the model for the interaction between defects.

\section{APPENDIX: COUPLING OF AN ELECTRIC FIELD WITH THE NEMATIC DIRECTOR FIELD IN THE VICINITY OF THE FREE INTERFACE}

As a useful simplification, the discussion here considers the structure of the director field to be planar, i.e., the escaped component along the interface is ignored. In the presence of an electric field, the bulk energy density will have the expression

$$
f=\frac{1}{2} K(\operatorname{grad} \Phi)^{2}-\frac{1}{2} \varepsilon_{0} \varepsilon_{\perp} E^{2}-\varepsilon_{0} \varepsilon_{a} E^{2} \sin ^{2}(\Phi),
$$

where $\varepsilon_{a}$ is the dielectric anisotropy of the medium $\left(\varepsilon_{a}\right.$ $\left.=\varepsilon_{\|}-\varepsilon_{\perp}\right)$. The director field is given by $\mathbf{n}$ $=[\sin (\Phi), 0, \cos (\Phi)]$. We consider the electric field to be applied by two parallel conductors placed at $z= \pm b / 2$ with a constant potential drop between them. The exact determination of the equilibrium director field configuration would require solving simultaneously the electrostatic equations coupled with the torque balance equation. As a first order approximation, we will consider a uniform electric field $\mathbf{E}$ $=E e_{z}$. This greatly simplifies the problem and allows us to derivate an equation for the director field by minimizing the energy density functional. The equations that describe the planar director field are

$$
\Delta \Phi+\frac{\varepsilon_{0} \varepsilon_{a} E^{2}}{2 K} \sin (2 \Phi)=0, \text { in the bulk, }
$$

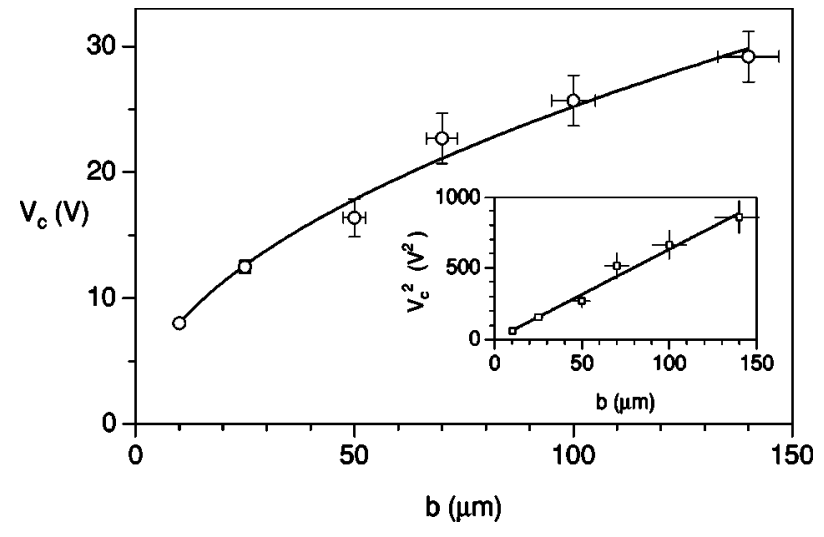

FIG. 11. The rms applied voltage above which the disclination line is believed to collapse, $V_{c}$, is represented as a function of sample thickness. The solid curve is a best fit to a square root. In the inset, the same data are represented as $V_{c}^{2}$ vs $b$, and the solid line is a linear fit to the data.

$$
K \frac{\partial \Phi}{\partial \nu}=\frac{W}{2} \sin (2 \varphi), \text { on the interface. }
$$

Here, $\varphi$ is the angle between the director field and the normal to the interface, and $\partial / \partial \nu$ is the derivative along that normal.

Dimensional analysis of these equations suggests that the functional dependence of the position of the disclination line, $d$, will be of the form

$$
d=b f\left(\frac{W b}{K}, \frac{\varepsilon_{0} \varepsilon_{a} V^{2}}{K}\right)
$$

where $V=E b$ is the applied voltage. For the cell gaps reported in this work, and for moderate voltages, we have observed that the position of the disclination line scales trivially with the sample thickness, i.e., $d / b$ appears to be independent of the cell gap. This suggests we are in the limit of strong anchoring on the free interface $(W b / K \gg 1)$, and $d / b$ $=f\left(\varepsilon_{0} \varepsilon_{a} V^{2} / K\right)$. By comparing measurements of $d / b$ performed under electric field for different liquid crystals, we have verified that the above relationship is consistent with the experiments. In particular, $V_{t h}$ (see Sec. IV C) appears to scale with the material properties as it is suggested by the above expression.

The sample thickness dependence in the position of the disclination line starts to appear at large applied voltages. In particular, we have observed that for a voltage above a thickness dependent value $V_{c}$ the disclination line appears to collapse. We have verified experimentally that $V_{c}$ scales as $b^{1 / 2}$ (see Fig. 11). This suggests that in this regime the position of the disclination line may have a functional dependence of the form $d=b f\left[\varepsilon_{0} \varepsilon_{a} V^{2} /(W b)\right]$. 
[1] E. Dzyaloshinskii, Zh. Éksp. Teor. Fiz. 31, 773 (1970) [Sov. Phys. JETP 33, 733 (1970)].

[2] P.E. Cladis and M. Kléman, J. Phys. (Paris) 33, 591 (1972).

[3] R.B. Meyer, Philos. Mag. 27, 405 (1973).

[4] C.E. Williams, P. Pieranski, and P.E. Cladis, Phys. Rev. Lett. 29, 90 (1972).

[5] C.E. Williams, P.E. Cladis, and Kléman, Mol. Cryst. Liq. Cryst. 21, 355 (1973).

[6] M. Kuzma and M. Labes, Mol. Cryst. Liq. Cryst. 100, 103 (1983).

[7] G.P. Crawford, M. Vilfran, J.W. Doane, and I. Vilfran, Phys. Rev. A 43, 835 (1991).

[8] I. Vilfan, M. Vilfan, and S. Zümer, Phys. Rev. A 43, 6875 (1991).

[9] G.P. Crawford and S. Zümer, Int. J. Mod. Phys. B 9, 331 (1995).

[10] G.G. Peroli and E.G. Virga, Phys. Rev. E 54, 5235 (1996).

[11] G.G. Peroli, G. Hillig, A. Saupe, and E.G. Virga, Phys. Rev. E 58, 3259 (1998).

[12] G.G. Peroli and E.G. Virga, Phys. Rev. E 59, 3027 (1999).
[13] G.P. Crawford, D.W. Allender, and J.W. Doane, Phys. Rev. A 44, 2570 (1991).

[14] T. Nagaya, H. Hotta, H. Orihara, and Y. Ishibashi, J. Phys. Soc. Jpn. 61, 3511 (1992).

[15] I. Chuang, B. Yurke, and A.N. Pargellis, Phys. Rev. E 47, 3343 (1993).

[16] J. Ignés-Mullol, J. Baudry, L. Lejcek, and P. Oswald, Phys. Rev. E 59, 568 (1999).

[17] M. Mihailovic and P. Oswald, J. Phys. (France) 49, 1467 (1988).

[18] J. Baudry, S. Pirkl, and P. Oswald, Phys. Rev. E 57, 3038 (1998).

[19] P.-G. de Gennes and J. Prost, The Physics of Liquid Crystals, 2nd ed. (Oxford University Press, New York, 1995).

[20] N.V. Madhusudana and R. Pratibha, Mol. Cryst. Liq. Cryst. 89, 249 (1982).

[21] A.D. Rutenberg and A.J. Bray, Phys. Rev. E 50, 1900 (1994).

[22] P. Biscari, G.G. Peroli, and E.G. Virga, Liq. Cryst. 26, 1825 (1999). 